\title{
REFRACTIVE INDICES OF SOLUTIONS OF HYDRO- CHLORIC ACID, ACETIC ACID AND ETHANOL IN WATER AT $25^{\circ}$ AND $30^{\circ}$
}

\author{
BY HOWARD M. ELSEY AND GEORGE L. LYNN*
}

The convenience of the dipping refractometer as a quick means of determining the concentration of solutions of pure substances in water is well known. However, its ready use in our laboratory for the analysis of solutions of hydrochloric acid, ${ }^{1}$ acetic acid, ${ }^{2}$ and ethano ${ }^{3}$ in water was hampered by the lack of sufficient data at the usual laboratory temperatures, the data of previous observers usually being taken at temperatures not conveniently maintained in many American laboratories, or else not covering the range of concentrations desired.

The hydrochloric acid and acetic acid solutions used in the following measurements were prepared from C. P. chemicals which were diluted with distilled water and the solutions aerated with clean air to remove carbon dioxide. The strength of these acid solutions was then determined by titrating a weighed amount of a solution with standard barium hydroxide. The strength of the barium hydroxide was determined by titrating

* Contribution from the Chemical Laboratory of the University of Kansas.

1 Willigen: Arch. Mus. Teyler, 2, 209 (1869); Schivers: Jour. Chim. phys., 8, 530 (1910); Schivers: Bull. Acad. roy. belg. (1910); Le Blanc: Zeit. phys. Chem., 4, 553 (1889); Wagner: Zeit. öffentl. Chem., 11, 404 (1905); Wagner: Tabellen zum Fintauchrefraktometer, Sondershausen (1903); Hallwachs: Wied. Ann., 53, 1 (1894).

2 Rimbach and Wintgen: Zeit. phys. Chem., 74, 233 (1910); Buchkreimer: Diss. Bonn (1890); Halwachs: Wied. Ann., 53, 1 (1894); Wagner: Tabellen zum Eintauchrefraktometer, Sondershausen (1903); Zeit. öffentl. Chem. 11, 404 (1905); LeBlanc: Zeit. phys. Chem., 4, 553 (1889) (R); Landolt: Pogg. Ann., 117,353 (1862).

Andrews: Jour. Amer. Chem. Soc., 30, 363 (1908); Schivers: Zeit. phys. Chem., 75, 357 (1911); Leech and Lythgoe: Jour. Am. Chem. Soc., 37, 954 (1905); Wagner: Tabellen zum Eintauchrefractometer. Sondershausen (1903); Zeit. öffentl. Chem., 11, 404 (1905); Hess: Wien. Ber, 114, 1231 (1905); Sidersky: Bull. assoc. chim. sucr. dist., 27, 1169 (1910). 
it against a hydrochloric acid solution which has been standardized by precipitation as silver chloride.

TABLE I

Refractive Indices of Water Solutions at $25^{\circ}$

\begin{tabular}{|c|c|c|c|c|c|}
\hline \multicolumn{3}{|c|}{ Hydrochloric Acid } & \multicolumn{3}{|c|}{ Hydrochloric Acid } \\
\hline $\mathrm{C}$ & $\mathrm{s}$ & $N_{D}$ & $\mathrm{C}$ & $\mathrm{s}$ & $\mathrm{N}_{\mathbf{D}}$ \\
\hline 160.3 & 98.06 & 1.36395 & 54.79 & 44.20 & $1 . \overline{34433}$ \\
\hline 148.6 & 92.17 & 1.36187 & 46.07 & 39.46 & 1.34255 \\
\hline 114.6 & 75.56 & 1.35590 & 36.92 & 34.32 & 1.34060 \\
\hline 103.3 & 69.62 & 1.35374 & 26.81 & 28.72 & 1.33847 \\
\hline 93.49 & 64.59 & 1.35190 & 17.72 & 23.57 & 1.33651 \\
\hline 84.67 & 60.10 & 1.35025 & 8.02 & 17.90 & 1. 33432 \\
\hline 73.81 & 54.46 & 1.34816 & 0.00 & 13.20 & 1. 33251 \\
\hline 65.90 & 50.32 & 1.34662 & & & \\
\hline \multicolumn{3}{|c|}{ Acetic Acid } & \multicolumn{3}{|c|}{ Acetic Acid } \\
\hline $\mathrm{C}$ & $S$ & $\mathrm{~N}_{\mathrm{D}}$ & $\mathrm{C}$ & $\mathrm{s}$ & $\mathrm{N}_{\mathrm{D}}$ \\
\hline 405.32 & 64.52 & 1.35187 & 179.87 & 40.86 & 1.34308 \\
\hline 407.12 & 65.56 & 1.35226 & 152.45 & 37.25 & 1.34172 \\
\hline 367.90 & 61.28 & 1.35068 & 120.41 & 32.76 & 1.34001 \\
\hline 333.44 & 58.06 & 1.34949 & 92.93 & 28.76 & 1. 33848 \\
\hline 303.94 & 55.05 & 1.34838 & 71.75 & 25.40 & 1.33720 \\
\hline 266.89 & 51.17 & 1.34694 & 47.42 & 21.46 & 1.33569 \\
\hline 239.65 & 48.11 & 1.34579 & 25.37 & 17.76 & 1.33427 \\
\hline 220.39 & 45.82 & 1.34493 & & & \\
\hline \multicolumn{3}{|c|}{ Ethanol } & \multicolumn{3}{|c|}{ Ethanol } \\
\hline $\mathrm{C}$ & S & $\mathrm{N}_{\mathrm{D}}$ & C & S & $N_{D}$ \\
\hline 397.12 & 62.75 & 1.35123 & 161.94 & 37.51 & 1.34181 \\
\hline 283.73 & 61.77 & 1.35086 & 129.24 & 32.76 & 1.34001 \\
\hline 341.34 & 58.37 & 1. 34961 & 104.72 & 29.17 & 1.33864 \\
\hline 301.30 & 54.52 & 1.34818 & 82.92 & 25.87 & 1.33739 \\
\hline 274.87 & 51.56 & 1.34709 & 59.46 & 22.35 & 1.33604 \\
\hline 241.17 & 47.75 & 1.34566 & 41.23 & 19.60 & 1.33498 \\
\hline 211.10 & 44.06 & 1.34428 & 24.93 & 17.11 & 1.33402 \\
\hline 181.05 & 40.07 & 1.34278 & & & - \\
\hline
\end{tabular}

The ninety-five percent grain alcohol used in preparing the ethanol solutions was diluted with water, filtered through magnesium carbonate, and then twice distilled, the final dis- 
tillate $^{1}$ being diluted with distilled water to prepare the samples.

TABLE II

Refractive Indices of Water Solutions at $30^{\circ}$

\begin{tabular}{|c|c|c|c|c|c|}
\hline \multicolumn{3}{|c|}{ Hydrochloric Acid } & \multicolumn{3}{|c|}{ Hydrochloric Acid } \\
\hline $\mathrm{C}$ & $\mathbf{S}$ & $\mathrm{N}_{\mathrm{D}}$ & $\mathrm{C}$ & $S$ & $N_{D}$ \\
\hline 160.3 & 96.37 & 1.36336 & 54.79 & 43.12 & 1.34393 \\
\hline 148.6 & 90.58 & 1.36130 & 46.07 & 38.25 & 1.34210 \\
\hline 114.6 & 74.00 & 1.35534 & 36.92 & 33.24 & 1.34019 \\
\hline 103.3 & 68.26 & 1.35325 & 26.81 & 27.52 & 1.33802 \\
\hline 93.49 & 63.33 & 1.35144 & 17.72 & 22.45 & 1.33608 \\
\hline 84.67 & 58.80 & 1.34977 & 8.02 & 16.76 & 1.33389 \\
\hline 73.81 & 53.20 & 1.34769 & 0.00 & 12.00 & 1.33204 \\
\hline 65.90 & 49.00 & 1.34613 & & & \\
\hline \multicolumn{3}{|c|}{ Acetic Acid } & \multicolumn{3}{|c|}{ Acetic Acid } \\
\hline C & $\mathrm{s}$ & $N_{D}$ & $\mathrm{C}$ & $\mathrm{S}$ & $N_{D}$ \\
\hline 405.32 & 61.52 & 1.35077 & 179.87 & 38.63 & 1.34223 \\
\hline 407.12 & 62.53 & 1.35115 & 152.45 & 35.17 & 1.34092 \\
\hline 367.90 & 58.47 & 1.34964 & 120.41 & 30.80 & 1. 33926 \\
\hline 333.44 & 55.31 & 1.34847 & 92.93 & 26.92 & 1.33779 \\
\hline 303.94 & 52.41 & 1.34740 & 71.75 & 23.73 & 1.33657 \\
\hline 266.89 & 48.62 & 1.34598 & 47.42 & 19.86 & 1.33509 \\
\hline 239.65 & 45.73 & 1.34490 & 25.37 & 16.18 & 1.33366 \\
\hline 220.39 & 43.44 & 1.34405 & & & \\
\hline \multicolumn{3}{|c|}{ Ethanol } & \multicolumn{3}{|c|}{ Ethanol } \\
\hline $\mathrm{C}$ & $\mathbf{S}$ & $\mathrm{N}_{\mathrm{D}}$ & $\mathrm{C}$ & $\mathbf{S}$ & $\mathrm{N}_{\mathrm{D}}$ \\
\hline 397.12 & 59.43 & 1.35000 & 161.94 & 35.65 & 1.34111 \\
\hline 283.73 & 58.72 & 1.34974 & 129.24 & 31.00 & 1.33934 \\
\hline 341.34 & 55.30 & 1.34847 & 104.72 & 27.65 & 1.33807 \\
\hline 301.30 & 51.82 & 1.34718 & 82.92 & 24.31 & 1.33679 \\
\hline 274.87 & 48.90 & 1.34609 & 59.46 & 20.94 & 1.33550 \\
\hline 241.17 & 45.35 & 1.34476 & 41.23 & 18.20 & 1.33444 \\
\hline 211.10 & 41.79 & 1.34342 & 24.93 & 15.76 & 1.33351 \\
\hline 181.05 & 38.10 & 1.34204 & & & \\
\hline
\end{tabular}

The concentrations of these ethanol solutions were then calculated from the densities. ${ }^{2}$ The accurate work on the 1 The ethanol thus obtained may have contained a trace of aldehydes and ketones.

\footnotetext{
2 Bureau of Standards Circular, No. 19.
} 
refractive indices of potassium chloride solutions at $25^{\circ}$ by Baxter and his students ${ }^{1}$ was utilized in checking the scale of the refractometer used and it was found to be correct.

The temperature of the solutions was kept constant to $\pm .05^{\circ}$ during the measurements by an automatically controlled thermostat.

The results obtained are given in Tables (I) and II) where $\mathrm{C}$ is the concentration of solute in grams per 1000 grams of water, $S$ is the scale reading of the refractometer and $N_{D}$ is refractive index for sodium light.

By plotting the above data, curves may be drawn from which a set of tables can be prepared. By reference to these tables, the concentration of a solution of any one of the three substances can be found by taking a single reading with the dipping refractometer at room temperature.

\section{Summary}

The refractive indices at $25^{\circ}$ and $30^{\circ}$ are given for a number of solutions of hydrochloric acid, acetic acid and ethanol in water.

Lawrence, Kansas

${ }^{1}$ Jour. Am. Chem. Soc., 33, 922 (1911). 